\title{
固体火箭发动机喷管烧蚀控制机制判别新方法
}

\author{
张斌", 刘宇, 王长辉, 任军学 \\ 北京航空航天大学宇航学院, 北京 100083 \\ * E-mail: zhangbin.1983@yahoo.com.cn \\ 收稿日期: 2009-11-21; 接受日期: 2010-06-17 \\ 国家高技术研究发展计划(“863”计划)(批准号: 2008AA7020508)资助项目
}

\begin{abstract}
摘要 合理的固体火箭发动机喷管烧蚀控制机制判别方法能提高烧蚀率计算的精度. 基于 $\mathrm{H}_{2} \mathrm{O}$ 和 $\mathrm{CO}_{2}$ 与 $\mathrm{C}$ 反应具有不同的化学反应常数, 综合考虑了壁面温度和燃气氧化组 分浓度对烧蚀率的影响, 提出了一种判别烧蚀控制机制的新方法. 应用该判别方法对喷 管喉忖烧蚀问题进行了数值模拟. 计算结果表明, 本文采用的计算方法得到的烧蚀率小 于按扩散速率控制和双控制计算得到的烧蚀率, 且与试验结果更接近. 同时指出燃气中 由 $\mathrm{H}_{2} \mathrm{O}$ 引起的烧蚀率是导致喷管喉忖烧蚀的主要原因.

关键词

喷管

烧蚀

扩散控制机制

化学反应控制机制

判别方法
\end{abstract}

目前, $\mathrm{C} / \mathrm{C}$ 复合材料以其一系列优异的性能成为 了固体火箭发动机喷管设计中广泛采用的烧蚀热防 护材料. $\mathrm{C} / \mathrm{C}$ 复合材料的烧蚀可分为热化学烧蚀和机 械烧蚀两部分. 前者指 $\mathrm{C}$ 的表面在高温气流环境下 和氧化组分 $\left(\mathrm{H}_{2} \mathrm{O}, \mathrm{CO}_{2}\right.$ 和 $\left.\mathrm{OH}\right)$ 发生的氧化反应; 后者 指气流压力和剪切力作用下因基体和纤维的密度不 同, 造成烧蚀差异而引起的颗粒状剥落或因热应力 破坏的片状剥落 ${ }^{[1,2]}$.

$\mathrm{C} / \mathrm{C}$ 复合材料的热化学烧蚀主要由以下两种因 素控制: 第一, 燃气中氧化组分向 $\mathrm{C}$ 表面的扩散速率; 第二, $\mathrm{C}$ 原子与燃气中氧化组分在高温条件下的化学 反应速率. 一般认为, 当 $\mathrm{C}$ 表面温度较高时, 化学反 应速率极快, 扩散到壁面的氧化组分很快被耗尽, 此 时烧蚀过程主要受燃气中氧化组分扩散速率控制; 相反, 当 $\mathrm{C}$ 表面温度较低时, 扩散过程极快, 化学反 应只能部分消耗扩散到壁面的氧化组分, 此时烧蚀 过程主要是由化学反应速率控制. 在喷管喉祄烧蚀 计算时, 由于其表面温度较高, 很多文献认为烧蚀过 程是由扩散速率控制的 ${ }^{[3,4]}$. 对于温度适中的情况文
献 $[5,6]$ 认为烧蚀由两者同时控制. 以上两种烧蚀判 别机制均提供了比较合理的烧蚀计算方法. 文献 [7] 对这两种判别方法提出了质疑. 该文认为这两种判 别烧蚀控制机制方法比较主观. 喷管烧蚀机制的判 别应由喷管内燃气中 $\mathrm{H}_{2}$ 的含量和 $\mathrm{H}_{2}$ 的活化能来决定. 本文赞同文献[7]这一观点, 仅根据 C 表面温度的高 低来选择烧蚀控制方式比较主观. 是否应该综合考 虑其他因素? 如燃气组分浓度等.

对于 $\mathrm{H}_{2}$ 与 $\mathrm{C}$ 反应一直有比较大的争论. 国外有 研究结果表明, 试验中得到 $\mathrm{C}$ 烧蚀率随着 $\mathrm{H}_{2}$ 组分浓 度的增加而减少 ${ }^{[8,9]}$. 所以基本能够否定该反应的发 生. 关于 $\mathrm{C}$ 与 $\mathrm{H}_{2} \mathrm{O}$ 和 $\mathrm{CO}_{2}$ 的反应目前国内外做过大 量研究. 其中 Libby 和 Blake 认为 ${ }^{[10]}$ 这两个反应的动 力学速度一样. 而国外有研究表明, 在高温下 $\mathrm{H}_{2} \mathrm{O}$ 更 容易和 $\mathrm{C}$ 反应 ${ }^{[11]}$. 因此这两个反应动力学速度是不 一样的.

本文在 $\mathrm{H}_{2} \mathrm{O}$ 和 $\mathrm{CO}_{2}$ 与 $\mathrm{C}$ 反应速率不同的基础上, 综合考虑了壁面温度和燃气氧化组分浓度对烧蚀率 的影响. 提出了更为合理的烧蚀控制机制判别方法, 
并通过烧蚀算例, 验证了该方法的合理性.

\section{1 控制机制的判别}

根据热力计算结果, 燃气组分随压强的变化很 小, 在发动机的工作压强范围内可以近似认为为常 数. 燃气中的主要组分为 $\mathrm{H}_{2} \mathrm{O}, \mathrm{CO}_{2}, \mathrm{HCl}, \mathrm{N}_{2}$ 和 $\mathrm{H}_{2}$, 此 外还含有少量的 $\mathrm{OH}$ 和 $\mathrm{H}$. 而 $\mathrm{O}$ 和 $\mathrm{O}_{2}$ 的成分由于燃 气基本属于富燃气体可以忽略. 对于含金属粒子的 推进剂产生的燃气还含有大量凝相 $\mathrm{Al}_{2} \mathrm{O}_{3}$. 一般假设 $\mathrm{HCl}, \mathrm{N}_{2}$ 和 $\mathrm{Al}_{2} \mathrm{O}_{3}$ 不与表面 C 发生反应. 烧蚀计算采用 的三方程热化学烧蚀模型如下:

$$
\left\{\begin{array}{l}
\mathrm{C}(\mathrm{s})+\mathrm{H}_{2} \mathrm{O} \rightarrow \mathrm{CO}+\mathrm{H}_{2} \\
\mathrm{C}(\mathrm{s})+\mathrm{CO}_{2} \rightarrow 2 \mathrm{CO} \\
\mathrm{C}(\mathrm{s})+\mathrm{OH} \rightarrow \mathrm{CO}+\mathrm{H}
\end{array}\right.
$$

由于 $\mathrm{OH}$ 的含量较少, 一般在进行烧蚀计算时也 可以忽略上述第三个反应. 此时为两方程的热化学 烧蚀模型. 在本文中, 化学反应动力学速率均采用了 Bradley 的数据 ${ }^{[11]}$. 因为这些参数已经被文献[12 14] 研究证明可靠.

由于 $\mathrm{H}_{2} \mathrm{O}$ 和 $\mathrm{CO}_{2}$ 与 $\mathrm{C}$ 反应速率不同, 本文将其 分开进行烧蚀计算. 假设 $\mathrm{H}_{2} \mathrm{O}$ 和 $\mathrm{CO}_{2}$ 与 $\mathrm{C}$ 反应是互 不干扰的. 例如, 在计算 $\mathrm{H}_{2} \mathrm{O}$ 引起的烧蚀率时, 认为 $\mathrm{CO}_{2}$ 不与 $\mathrm{C}$ 反应. 比较其化学反应烧蚀率与扩散烧蚀 率的大小, 取其小者为主要烧蚀率

$$
r_{\mathrm{H}_{2} \mathrm{O}}=\min \left(r_{\mathrm{H}_{2} \mathrm{O} \text {-diff }}, r_{\mathrm{H}_{2} \mathrm{O} \text {-chem }}\right) \text {. }
$$

采用相同的方法可以计算出 $\mathrm{CO}_{2}$ 引起的烧蚀率

$$
r_{\mathrm{CO}_{2}}=\min \left(r_{\mathrm{CO}_{2} \text {-diff }}, r_{\mathrm{CO}_{2} \text {-chem }}\right) \text {. }
$$

最后将两者相加得到总的烧蚀率

$$
r=r_{\mathrm{CO}_{2}}+r_{\mathrm{H}_{2} \mathrm{O}},
$$

其中下标 diff 和 chem 分别表示扩散控制和化学反应 控制, $r$ 为线烧蚀率.

\section{2 烧蚀模型}

\section{1 化学动力控制下 $\mathrm{C} / \mathrm{C}$ 材料线烧蚀速率计算}

\subsection{1 质量守恒方程.}

由组元 $i$ 在壁面组元的连续方程可推导出下式:

$$
\left(1+B^{\prime}\right) P_{i w}=P_{i e}+\beta w_{i},(i=1,2, \cdots, 7) .
$$

将(5)式应用到各气体组分中可得:

$$
\begin{gathered}
\left(1+B^{\prime}\right) P_{1 w}=P_{1 e}-\beta w_{1}, \\
\left(1+B^{\prime}\right) P_{2 w}=P_{2 e}-\beta w_{2}, \\
\left(1+B^{\prime}\right) P_{3 w}=P_{3 e}-\beta w_{3}, \\
\left(1+B^{\prime}\right) P_{4 w}=P_{4 e}+\frac{M_{4}}{M_{1}} \beta w_{1}+2 \frac{M_{4}}{M_{2}} \beta w_{2}+\frac{M_{4}}{M_{3}} \beta w_{3}, \\
\left(1+B^{\prime}\right) P_{5 w}=P_{5 e}+\frac{M_{5}}{M_{1}} \beta w_{1}, \\
\left(1+B^{\prime}\right) P_{6 w}=P_{6 e}+\frac{M_{6}}{M_{3}} \beta w_{3}, \\
\left(1+B^{\prime}\right) P_{7 w}=P_{7 e} .
\end{gathered}
$$

总体质量守恒为

$$
B^{\prime}=\beta M_{c}\left(\frac{w_{1}}{M_{1}}+\frac{w_{2}}{M_{2}}+\frac{w_{3}}{M_{3}}\right) .
$$

化学反应控制下 $\mathrm{C} / \mathrm{C}$ 材料线烧蚀率计算公式为

$$
\dot{r}_{\text {chem }}=\dot{m}_{c} / \rho_{c}=B^{\prime} /\left(\beta \rho_{c}\right),
$$

其中下标 $w$ 和 $e$ 分别表示壁面和外缘. $P$ 和 $M$ 分别表 示组分所占比例和组分分子量. $W$ 表示质量生成率. $B^{\prime}$ 和 $\beta$ 为两个无因次参数. 将不参加化学反应组分等 效折算到 $\mathrm{N}_{2} . i$ 分别表示组分: $\mathrm{H}_{2} \mathrm{O}, \mathrm{CO}_{2}, \mathrm{OH}, \mathrm{CO}, \mathrm{H}_{2}$, $\mathrm{H}, \mathrm{N}_{2}$.

\subsection{2 化学反应控制方程}

化学动力学控制的反应速率由阿累尼乌斯公式 给出:

$$
k_{i}=A_{i} T_{w}^{b} \exp \left(-E_{i} /\left(R T_{w}\right)\right),
$$

其中 $A_{i}$ 和 $E_{i}$ 分别为 $\mathrm{C}$ 与 $i$ 组份反应的频率因子和活 化能. $R, T_{w}$ 分别表示摩尔气体常数和喉祄表面温度. 表 1 列出了这三个反应的相关化学动力学参数.

\subsection{3 能量守恒方程}

根据能量守恒原理, 烧蚀表面能量转换处于动 态平衡, 有:

$$
\begin{gathered}
\dot{q}_{\mathrm{int}}=q_{\mathrm{conv}}+\dot{q}_{\mathrm{rad}}-\dot{q}_{\mathrm{chem}}, \\
\dot{q}_{\mathrm{conv}}=h_{c}\left(T_{e}-T_{w}\right),
\end{gathered}
$$

\section{表 1 相关化学动力学参数}

\begin{tabular}{lcccc}
\hline Surface reaction & $A_{i}\left(\mathrm{~kg} / \mathrm{m}^{2} / \mathrm{s}\right)$ & $b$ & $E_{i}(\mathrm{kcal} / \mathrm{mol})$ & $W_{i}\left(\mathrm{~kg} / \mathrm{m}^{2} / \mathrm{s}\right)$ \\
\hline $\mathrm{C}(\mathrm{s})+\mathrm{H}_{2} \mathrm{O} \rightarrow \mathrm{CO}+\mathrm{H}_{2}$ & $4.8 \times 10^{5}$ & 0 & 68.8 & $k_{i} P_{\mathrm{H}_{2}}{ }^{0.5}$ \\
$\mathrm{C}(\mathrm{s})+\mathrm{CO}_{2} \rightarrow 2 \mathrm{CO}$ & $9.0 \times 10^{3}$ & 0 & 68.1 & $k_{i} P_{\mathrm{CO}_{2}}{ }^{0.5}$ \\
$\mathrm{C}(\mathrm{s})+\mathrm{OH} \rightarrow \mathrm{CO}+\mathrm{H}$ & $3.61 \times 10^{2}$ & -0.5 & 0.00 & $k_{i} P_{\mathrm{OH}}$ \\
\hline
\end{tabular}




$$
\begin{aligned}
& \dot{q}_{\mathrm{rad}}=\varepsilon_{\mathrm{eff}} \sigma\left(T_{e}^{4}-T_{w}^{4}\right), \\
& \dot{q}_{\mathrm{chem}}=\sum_{i=1}^{3} \frac{\dot{w}_{i}}{M_{i}} \cdot \Delta H_{t},
\end{aligned}
$$

其中 $\dot{q}_{\text {int }}, q_{\text {conv }}, \dot{q}_{\text {rad }}, \dot{q}_{\text {chem }}$ 分别表示净热流率、对流热 流率、净辐射热流率、化学反应热流率. $h_{c}$ 表示对流 换热系数. $T_{e}$ 表示燃气温度. $\Delta H_{i}$ 表示 $\mathrm{C}$ 与 $i$ 组元反应 的标准生成焓. $\varepsilon_{\text {eff }}$ 表示喉祄材料的表面辐射因子。 $\sigma$ 表示 Steffens 常数.

\subsection{4 热传导方程}

一维轴对称热传导方程为

$$
\frac{\rho_{c} c}{\lambda_{c}} \frac{\partial T}{\partial t}=\frac{\partial^{2} T}{\partial r^{2}}+\frac{1}{r} \frac{\partial T}{\partial r} .
$$

初始边界条件: $T(r)=T_{0}$,

内壁边界条件: $-\lambda \frac{\partial T}{\partial r}=q_{\mathrm{int}}$,

外壁边界条件: $-\lambda \frac{\partial T}{\partial r}=0$ ，

其中 $\rho_{c}, \lambda_{c}$ 分别表示喉祄材料的密度、热容和导热系 数. 具体热传导方程计算方法见文献[15].

\subsection{5 计算流程}

计算流程大致可分为以下几个步骤：第一, 在已 知初始条件和边界条件情况下, 通过求解热传导方 程获得到 $t=n$ 时刻温度场分布和壁面温度; 第二, 将 壁面温度带入质量守恒方程和化学反应控制方程求 出 $t=n$ 时刻内的线烧蚀率和净热流率; 第三, 将净热 流率带入热传导方程获得 $t=n+1$ 时刻的壁面温度.

\section{2 扩散控制下 $\mathrm{C} / \mathrm{C}$ 材料线烧蚀速率计算}

根据扩散理论和化学动力学理论, 扩散控制下 碳/碳材料线烧蚀率为

$$
r_{\text {diff }}=\frac{h_{c}\left(\frac{M_{\mathrm{C}}}{M_{\mathrm{H}_{2} \mathrm{O}}} P_{1 e}+\frac{M_{\mathrm{C}}}{M_{\mathrm{CO}_{2}}} P_{2 e}+\frac{M_{\mathrm{C}}}{M_{\mathrm{OH}}} P_{3 e}\right)}{C_{p} \rho_{c}},
$$

其中 $C_{p}$ 表示燃气的比热容.

\section{3 算例分析}

3.1 和 3.2 算例计算所用参数为: 燃烧室平均压 强为 $4.9 \mathrm{MPa}$, 喉衬密度和厚度分别为 $1900 \mathrm{~kg} / \mathrm{m}^{3}$ 和
$80 \mathrm{~mm}$ ，喷管喉部直径 $25.4 \mathrm{~mm}$.

\section{1 由 $\mathrm{H}_{2} \mathrm{O}$ 引起的烧蚀速率计算分析}

已知然气中 $\mathrm{H}_{2} \mathrm{O}$ 的浓度为 $15 \%$, 燃气温度分别 为 $3200,3500,3800 \mathrm{~K}$. 计算结果见图 1. 如图 1 所示, 在开始烧蚀的时候, 热化学烧蚀率变化趋势为先增 大，后趋于稳定. 而扩散烧蚀率变化趋势为先减小, 后趋于稳定. 化学烧蚀率随温度的增加有明显的增 大. 这是因为温度对化学反应速率有很大影响. 而扩 散烧蚀率随温度的增加略有减小. 根据公式(9)可知, 在其他条件不变的情况下, 由于对流换热系数随着 燃气温度增加会略有下降，这使得扩散速率也有所 下降. 当温度为 3200 和 $3500 \mathrm{~K}$ 时，在计算时间内， 扩散烧蚀率均大于热化学烧蚀率. 而当温度为 3800 $\mathrm{K}$ 时, 热化学烧蚀率大约在 $T=8 \mathrm{~s}$ 时赶超扩散烧蚀率. 在计算初期, 壁面温度较低, 烧蚀主要由化学反应速 率控制, 随着壁面温度逐渐增加, 烧蚀控制机制可能 由化学反应速率控制转为扩散速率控制.

假设燃气温度为 $3500 \mathrm{~K}$, 燃气中 $\mathrm{H}_{2} \mathrm{O}$ 的浓度分 别为 $5 \%, 15 \%, 25 \%$. 计算结果见图 2. 分析图 2 可知, 扩散烧蚀和化学烧蚀均随浓度的增加而明显增加. 当 $\mathrm{H}_{2} \mathrm{O}$ 浓度为 $5 \%$ 情况下, 化学烧蚀率在大约 $4 \mathrm{~s}$ 时 赶超扩散烧蚀率. 而其他两种浓度情况下, 在计算时 间内, 扩散烧蚀率均大于热化学烧蚀率. 这说明随着 $\mathrm{H}_{2} \mathrm{O}$ 浓度的增加, $\mathrm{H}_{2} \mathrm{O}$ 与 $\mathrm{C}$ 反应引起的热化学烧蚀率 越难赶超扩散烧蚀率。

综上所述，对于燃气中 $\mathrm{H}_{2} \mathrm{O}$ 来说，应综合考虑壁

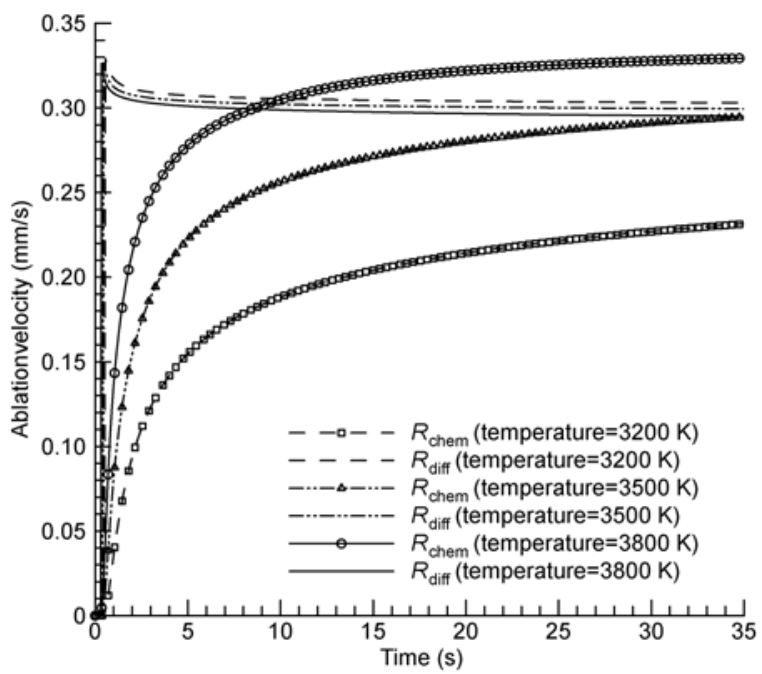

图 1 不同温度下烧蚀率随时间变化曲线 $\left(\mathrm{H}_{2} \mathrm{O}\right.$ 引起 $)$ 


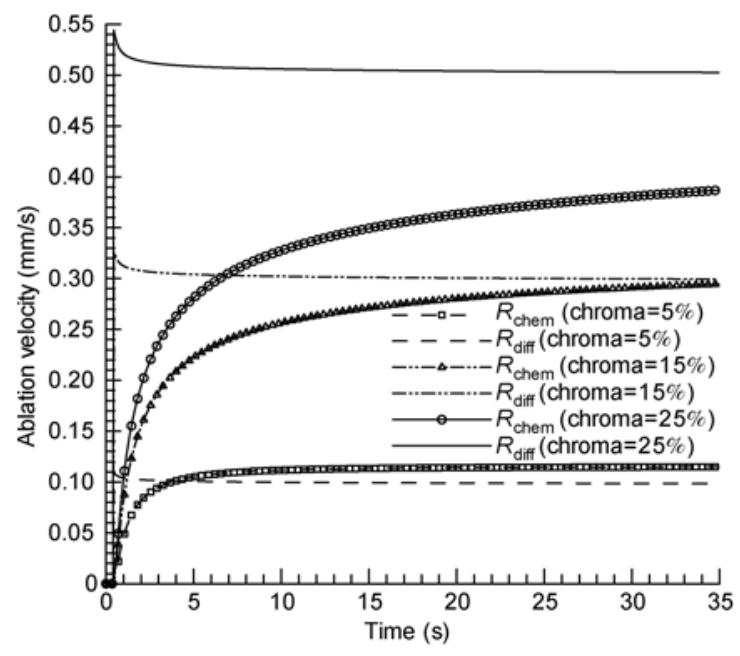

图 2 不同浓度下烧蚀率随时间变化曲线 $\left(\mathrm{H}_{2} \mathrm{O}\right.$ 引起 $)$

面温度和燃气中 $\mathrm{H}_{2} \mathrm{O}$ 组分浓度对烧蚀率的影响, 以 此来选择合理的烧蚀控制机制.

\section{2 由 $\mathrm{CO}_{2}$ 引起的烧蚀速率计算分析}

假设燃气中 $\mathrm{CO}_{2}$ 的浓度为 $15 \%$, 燃气温度分别 为 3200, 3500, $3800 \mathrm{~K}$. 计算结果见图 3. 图 3 中化学 反应烧蚀率和扩散烧蚀率的变化趋势与图 1 相似. 与 图 1 不同的是扩散烧蚀率远大于热化学烧蚀率. 比较 图 1 和图 3 的化学烧蚀率, 可以发现 $\mathrm{CO}_{2}$ 与 $\mathrm{C}$ 反应的 速率远小于 $\mathrm{H}_{2} \mathrm{O}$ 与 $\mathrm{C}$ 反应的速率. 这是因为 $\mathrm{H}_{2} \mathrm{O}$ 和 $\mathrm{CO}_{2}$ 具有不同的反应活化能和指前因子. 这说明 $\mathrm{H}_{2} \mathrm{O}$ 在高温高压下更容易和 $\mathrm{C}$ 反应.

假设燃气温度为 $3500 \mathrm{~K}$, 燃气中 $\mathrm{CO}_{2}$ 的浓度分

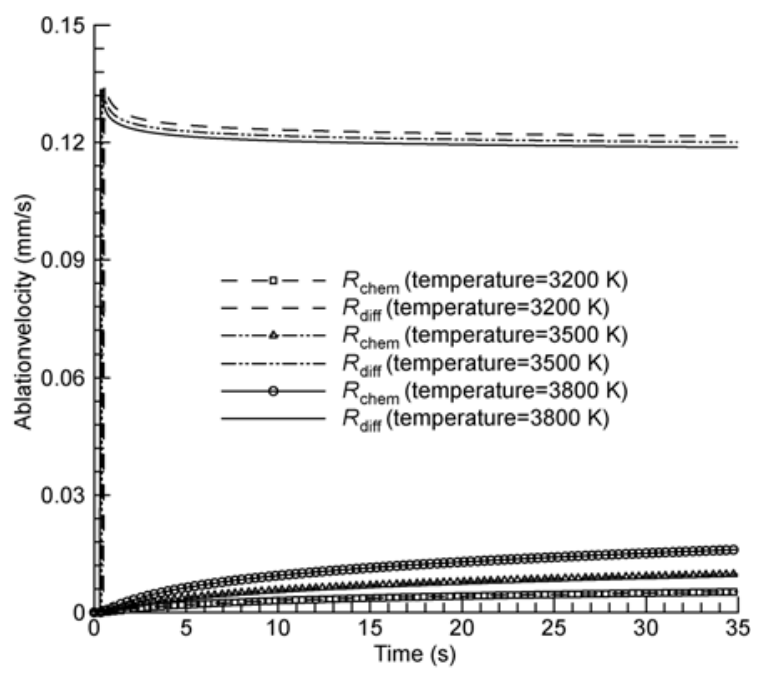

图 3 不同温度下烧蚀率随时间变化曲线 $\left(\mathrm{CO}_{2}\right.$ 引起 $)$
别为 5\%,15\%,25\%.计算结果见图 4. 从图 4 可知. 浓度对 $\mathrm{CO}_{2}$ 引起的扩散烧蚀率影响较大, 而对化学 烧蚀率影响较小. 浓度越大, 化学烧蚀率与扩散烧蚀 率差距越大. 比较图 2 和图 4 可知. $\mathrm{H}_{2} \mathrm{O}$ 引起的扩散 烧蚀率比 $\mathrm{CO}_{2}$ 引起的烧蚀率要大约 2.5 倍. 这是公式 (9) 中有 $M_{\mathrm{CO}_{2}} / M_{\mathrm{H}_{2} \mathrm{O}} \approx 2.5$, 同时其他相关参数, 如 $h_{c}$, $C_{p}, \rho_{c}, K_{1 e}, K_{2 e}$, 在任意时刻均相同.

综上所述, 由于由 $\mathrm{CO}_{2}$ 引起的化学烧蚀远小于 扩散烧蚀, 在喷管喉部烧蚀计算时, 由 $\mathrm{CO}_{2}$ 引起的烧 蚀率可全部采用化学反应速率控制.

\section{3 烧蚀算例}

分别采用扩散速率控制模型(Model-1)、双控制 模型(Model-2)、本文模型(current model)对文献[16] 中的 5 个算例进行烧蚀计算. BATES(Ballistics test and Evaluation System)发动机被广泛用于研究固体火 箭发动机喷管材料的烧蚀过程. Geisler 测得该发动机 喷管出现烧蚀大约是 1 2 $\mathrm{s}$ 之间. 这是因为该发动机 从点火到稳定工作状态大约需要 $1 \mathrm{~s}$, 导致喷管出现烧 蚀的时间有所延迟. 由于本文在计算开始时发动机已 经处于稳定状态，所以没有这段延迟时间出现．计算 所需参数有: 喉祄密度 $1830 \mathrm{~kg} / \mathrm{m}^{3}$, 喉部直径 $25.4 \mathrm{~mm}$, 喉衬厚度 $80 \mathrm{~mm}$. 其他计算所用参数见表 2 .

图 5 和图 6 分别给出了按本文模型计算前两个算 例中由 $\mathrm{H}_{2} \mathrm{O}$ 和 $\mathrm{CO}_{2}$ 引起烧蚀率. 如图 6 所示, 这两个 算例中, $\mathrm{CO}_{2}$ 扩散烧蚀率均远大于化学烧蚀率. 所以 $\mathrm{CO}_{2}$ 引起的烧蚀速率均由化学反应速率控制. 算例 1

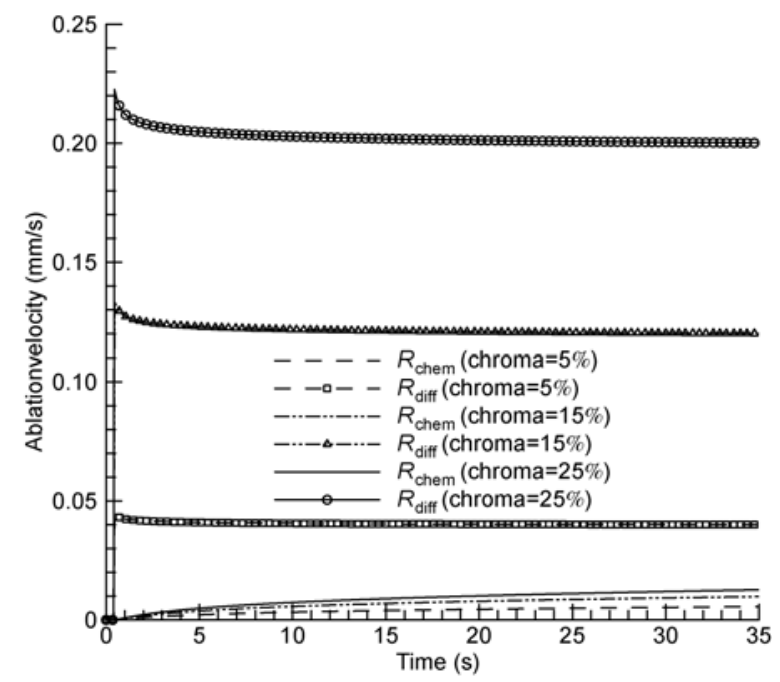

图 4 不同浓度下烧蚀率随时间变化曲线 $\left(\mathrm{CO}_{2}\right.$ 引起) 
表 2 Geisler 算例部分计算所用参数

\begin{tabular}{lccccc}
\hline \multicolumn{1}{c}{ Case } & Case 1 & Case 2 & Case 3 & Case 4 & Case 5 \\
\hline$P(\mathrm{MPa})$ & 6.9 & 6.9 & 6.9 & 6.9 & 6.9 \\
$T(\mathrm{~K})$ & 3580 & 3655 & 3715 & 3750 & 3745 \\
$\mathrm{Al}$ & $15 \%$ & $18 \%$ & $21 \%$ & $24 \%$ & $27 \%$ \\
$Y_{\mathrm{CO}_{2}}$ & 0.04 & 0.025 & 0.015 & 0.005 & 0.0015 \\
$Y_{\mathrm{H}_{2} \mathrm{O}}$ & 0.145 & 0.105 & 0.07 & 0.045 & 0.025 \\
$Y_{\mathrm{H}_{2}}$ & 0.02 & 0.02 & 0.02 & 0.02 & 0.02 \\
$Y_{\mathrm{HCL}}$ & 0.24 & 0.23 & 0.195 & 0.190 & 0.190 \\
$Y_{\mathrm{AL}_{2} \mathrm{O}_{3}}$ & 0.28 & 0.34 & 0.40 & 0.44 & 0.47 \\
\hline
\end{tabular}

中, $\mathrm{H}_{2} \mathrm{O}$ 扩散烧蚀率同样大于化学烧蚀率. 所以在计 算时间内烧蚀速率始终受化学反应速率控制. 而在 算例 2 中, $\mathrm{H}_{2} \mathrm{O}$ 化学烧蚀率不断追赶扩散烧蚀率, 大 约在 $3.2 \mathrm{~s}$ 时化学烧蚀率赶超扩散烧蚀率. 所以在 3.2 $\mathrm{s}$ 前为化学反应速率控制; $3.2 \mathrm{~s}$ 之后为扩散速率控制. 算例 3 至算例 5 选择的烧蚀控制机制与算例 2 相同. 同时, 比较图 5 和图 6 烧蚀率, 可以发现算例中 $\mathrm{H}_{2} \mathrm{O}$ 引起的烧蚀率远大于 $\mathrm{CO}_{2}$ 引起的烧蚀率.

图 7 给出了采用本文模型计算不同算例下净烧 蚀率随时间变化曲线. 如图 7 所示, 各算例烧蚀率变 化趋势近似线形. 算例 1 至算例 5 的烧蚀率逐渐下降, 这是因为随着 $\mathrm{Al}$ 含量的增加, $\mathrm{H}_{2} \mathrm{O}$ 和 $\mathrm{CO}_{2}$ 的浓度逐 渐减少, 这导致了喉忖 $\mathrm{C}$ 烧蚀率逐渐减小.

三个模型计算值和试验值对比见表 3. 分析表 3 可知, 与其他双控模型和本文提出模型计算结果相

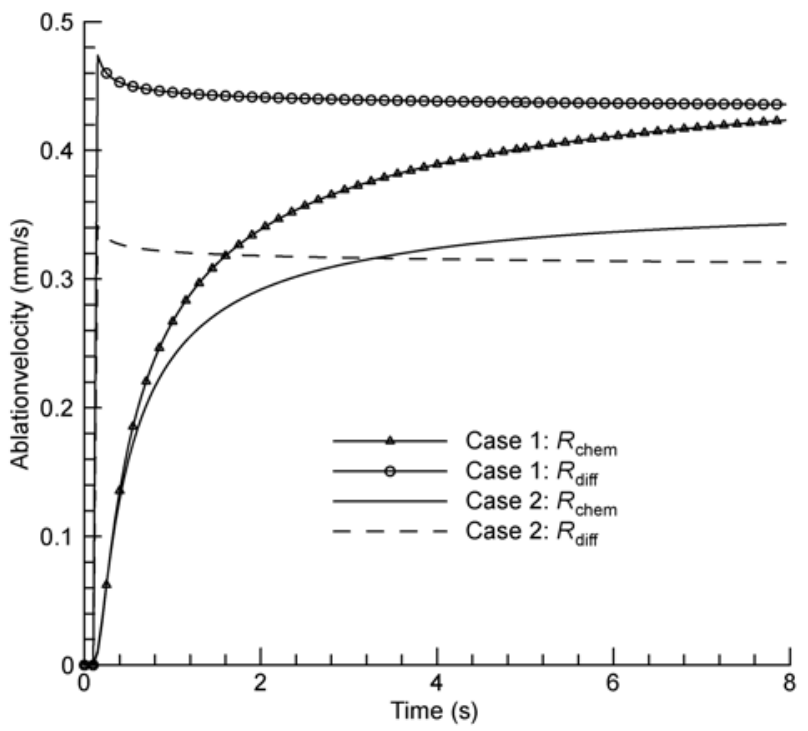

图 5 算例 1 2 计算烧蚀率随时间变化曲线 $\left(\mathrm{H}_{2} \mathrm{O}\right.$ 引起)

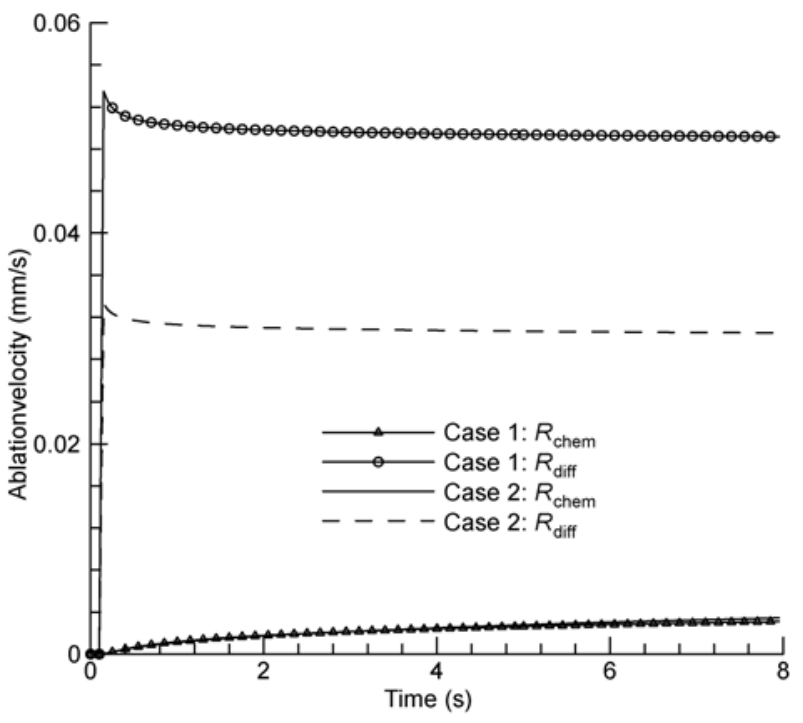

图 6 算例 1 2 计算烧蚀率随时间变化曲线 $\left(\mathrm{CO}_{2}\right.$ 引起)

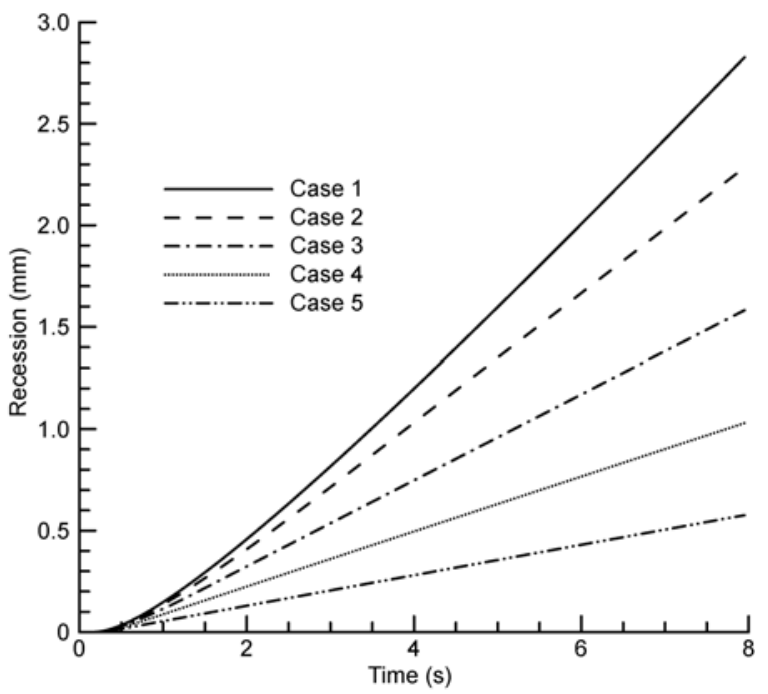

图 7 不同算例烧蚀厚度随时间的变化曲线

表 3 计算平均烧蚀率与试验对比

\begin{tabular}{lcccc}
\hline & Model 1 & Model 2 & Current model & Exp \\
\hline Case 1 & 0.493 & 0.363 & 0.362 & 0.353 \\
Err & $40.0 \%$ & $2.8 \%$ & $2.5 \%$ & - \\
Case 2 & 0.349 & 0.302 & 0.292 & 0.284 \\
Err & $22.9 \%$ & $6.3 \%$ & $2.8 \%$ & - \\
Case 3 & 0.229 & 0.214 & 0.202 & 0.200 \\
Err & $14.5 \%$ & $7.0 \%$ & $1.0 \%$ & - \\
Case 4 & 0.141 & 0.135 & 0.131 & 0.124 \\
Err & $13.7 \%$ & $8.9 \%$ & $5.6 \%$ & - \\
Case 5 & 0.077 & 0.075 & 0.073 & 0.069 \\
Err & $11.6 \%$ & $8.7 \%$ & $5.8 \%$ & - \\
\hline
\end{tabular}


比，按扩散速率控制模型计算结果误差大于其他两 种控制机制计算结果误差. 随着 $\mathrm{Al}$ 含量的增加计算 误差逐渐减小. 这种变化趋势可以通过分析各算例 中由 $\mathrm{H}_{2} \mathrm{O}$ 和 $\mathrm{CO}_{2}$ 引起烧蚀得到解释. 算例 1 中 $\mathrm{H}_{2} \mathrm{O}$ 和 $\mathrm{CO}_{2}$ 引起的烧蚀率本应由化学反应速率控制, 而 此时采用扩散速率控制计算会导致较大误差. 其他 四个算例中由 $\mathrm{H}_{2} \mathrm{O}$ 引起的烧蚀率均出现了由化学反 应速率控制转至扩散速率控制. 且控制方式转换出 现时间逐渐前移(算例 2 至算例 5 种控制方式开始转 变的时间分别为 $3.2,1.5,0.9,0.6 \mathrm{~s}$ ).

双控制模型和本文模型计算结果与试验值较接 近. 算例 1 中双控制模型和本文模型均采用化学反应 速率控制, 所以计算值基本一致. 而其他 4 个算例中, 当采用扩散速率控制时, 由 $\mathrm{CO}_{2}$ 引起的烧蚀率被夸 大了. 但由于 $\mathrm{CO}_{2}$ 引起的烧蚀率量级较小, 所以双控 模型和本文模型计算值相差不大. 算例 2 至算例 5 扩 散速率控制所占的时间比例越大, 所以按双控制模 型计算结果误差逐渐增大. 而按本文模型计算时则 不出现以上问题.

\section{4 结论}

1) 提出了一种喷管烧蚀控制机制判别新方法, 具体表示如下:

$r=\min \left(r_{\mathrm{H}_{2} \mathrm{O} \text {-diff }}, r_{\mathrm{H}_{2} \mathrm{O} \text {-chem }}\right)+\min \left(r_{\mathrm{CO}_{2} \text {-diff }}, r_{\mathrm{CO}_{2} \text {-chem }}\right)$.

2) 计算 $\mathrm{H}_{2} \mathrm{O}$ 引起的烧蚀率时，应综合考虑壁面 温度和燃气组分中 $\mathrm{H}_{2} \mathrm{O}$ 浓度来选择烧蚀控制机制. 而计算 $\mathrm{CO}_{2}$ 引起的烧蚀率时, 可选用化学反应速率 控制.

3) 在喷管喉忖烧蚀计算时, 由 $\mathrm{H}_{2} \mathrm{O}$ 引起的烧蚀 率远大于 $\mathrm{CO}_{2}$ 引起的烧蚀率, 所以 $\mathrm{H}_{2} \mathrm{O}$ 引起的烧蚀 率是导致喉忖烧蚀的主要因.

4) 通过应用扩散速率控制模型、双控制模型和 本文模型计算 Geisler 的 5 个算例. 发现扩散速率控 制模型计算结果误差较大，而双控制模型和本文模 型计算结果与试验值相似.

5) 随着 $\mathrm{Al}$ 含量的增加, 扩散速率控制模型结算 结果误差逐渐减小，双控制模型计算结果误差逐渐 增大. 而本文模型不存在这类问题.

\section{参考文献}

1 黄海明, 杜善义, 吴林志, 等. C/C 复合材料烧蚀性能分析. 复合材料学报, 2001, 18: 76-80

2 尹健，熊湘，张红波，等. 固体火箭发动机喷管用 $\mathrm{C} / \mathrm{C}$ 复合材料的研究进展. 材料导报, 2004, 18: 46-48

3 方丁酉, 夏智勋, 姜春林. C/C 喉忖稳态烧蚀的工程计算. 固体火箭技术, 2000, 23: 24-27

4 何洪庆, 周旭. 固体火箭喷管中的烧蚀控制机制. 推进技术, 1993, 4: 36-41

5 Kuo K K, Keswani S T. A comprehensive theoretical model for carbon-carbon composite nozzle recession. Combust Sci Tech, 1986, 42: $177-192$

6 Acharya R, Kuo K K. Effect of chamber pressure and propellant composition on erosion rate of graphite rocket nozzle. J Propul Power, 2007, 23: 1242-1254

7 黄海明，徐晓亮，姜贵庆. 固体火箭喷管烧蚀控制机制的判别. 中国科学 E 辑：中国科学, 2009, 39: 1558-1563

8 Borie V, Brulard J, Lengelle G. Aerothermochemical analysis of carbon-carbon nozzle regression in solid-propellant rocket motors. J Propul Power, 1989, 56: 65-73

9 Klager K. The interaction of the efflux of solid propellants with nozzle materials. Propell Explos, 1977, 2: 55—63

10 Libby P A, Blank T R. Buring carbon particles in the presence of water vapor. Combus Flame, 1981, 141: 123-147

11 Bradley D, Dixon-Lewis G, Habik E S, et al. The oxidation of graphite powder in flame reacion zones. In: 20th Souposium (International) on Combustion. Piusburgh, PA, 1984. 931-940

12 Piyush T, Vigor Y. Chemical erosion of carbon-carbon/graphite nozzles in solid-propellant rocket motors. J Propul Power, 2008, 24: $822-833$

13 Daniele B, Francesco N, Emanuele M. Coupled analysis of flow and surface ablation in carbon-carbon rocket nozzles. J Space Rocket, 2009, 46: 492-500

14 Piyush T, Vigor Y. Mitigation of graphite nozzle erosion by boundary-layer control in solid rocket motors. J Propul Power, 2009, 25: $1079-1085$

15 蔡体敏. 固体火箭发动机工作过程的数值模拟. 西安: 西北工业大学出版社, 1990

16 Geisler R L. The relationship between solid propellant formulation variables and nozzle recession rates. JANNAF Rocket Nozzle Technology Subcommittee Meeting. Lancaster, CA, 1978 